\title{
Cognitive therapy reduced relapses and number of days in bipolar episodes in DSM-IV bipolar I disorder
}

Lam DH, Hayward P, Watkins ER, et al. Relapse prevention in patients with bipolar disorder: cognitive therapy outcome after 2 years. Am J Psychiatry 2005; 162:324-9.

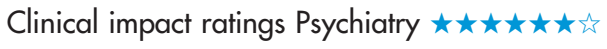

In patients with DSM-IV bipolar I disorder, is medication plus cognitive therapy (CT) more effective than medication alone for reducing relapses and number of days in bipolar episodes?

\section{METHODS}

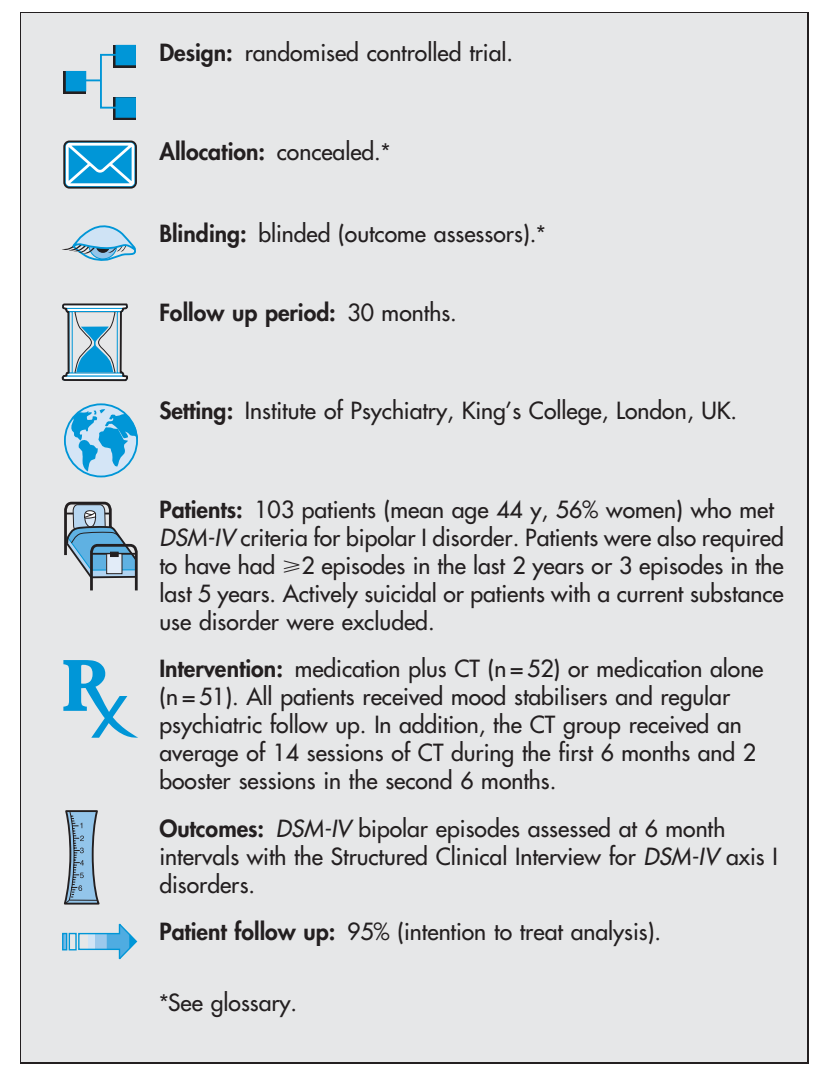

\section{MAIN RESULTS}

Over the 30 months, about a quarter fewer relapses were observed in the CT group than in the control group (table); the mean number of days in an illness episode was lower (difference -110.5 d, 95\%

For correspondence: Dr D H Lam, King̀s College, London, UK. spitdh|@iop. kcl.ac.uk

Source of funding: not stated
CI -188.9 to -32.1 ). During the last 18 months, mean number of days in an illness episode was lower in the CT group than in the control group (difference $-53.7 \mathrm{~d}$, CI -104.7 to -2.8 ), but the groups did not differ for relapse rate (table).

\section{CONCLUSION}

In patients with $D S M-I V$ bipolar I disorder, medication plus cognitive therapy was more effective than medication alone for reducing relapses and number of days in bipolar episodes.

\section{Commentary}

These findings by Lam et al contribute to the fledgling literature showing that psychosocial interventions contribute to the treatment of bipolar disorder. The conclusion that adding CT to medication reduces relapse is clinically significant for several reasons. Medications alone do not prevent relapse; up to $73 \%$ of patients with bipolar disorder who adhere to medications relapse in the 5 years after an episode. ${ }^{1}$ Each episode of a mood disorder appears to reduce the threshold intensity of the stressor that is required to trigger a subsequent episode ${ }^{2}$ and result in increased impairment.

These findings deserve careful attention from clinicians who treat bipolar disorder, because many of the interventions in the CT (detailed in

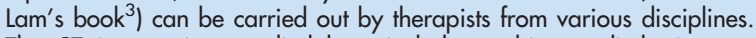
The CT interventions studied here include teaching a diathesis-stress model that emphasises the need for combined medication and psychological interventions; teaching patients to monitor prodromes and change behaviour to prevent prodromes from developing into full blown episodes; promoting regular sleep and routine; and helping patients modulate extreme striving to achieve unrealistically high goals. ${ }^{4}$

$$
\text { Jacqueline B Persons, PhD }
$$
San Francisco Bay Area Center for Cognitive Therapy Oakland, California, USA University of California-Berkeley, Berkeley, California, USA June L Gruber, MA

University of California-Berkeley, Berkeley, California, USA

1 Gitlin M, Swendsen J, Heller TL, et al. Relapse and impairment in bipolar disorder. Am J Psychiatry 1995; 152:1635-40.

2 Post RM. Transduction of psychosocial stress into the neurobiology of recurrent affective disorder. Am J Psychiatry 1992;149:999-1010.

3 Lam D. Cognitive therapy for bipolar disorder: a therapist's guide to concepts, methods, and practice. New York: Wiley, 1999.

4 Johnson SL. Mania and dysregulation in goal pursuit: a review. Clin Psychol Rev 2005;25:241-62.

Medication plus cognitive therapy (CT group) $v$ medication alone (control group) in DSM-IV bipolar I disorder*

\begin{tabular}{llllll}
\hline Outcomes at & Follow up period & CT group & Control group & RRR (95\% CI) & NNT (CI) \\
\hline Patients with $\geqslant 1$ & Months 1-30 & $63.8 \%$ & $84.3 \%$ & $24.3 \%(4.3$ to 42.4$)$ & 5 (3 to 32) \\
relapse & Months 13-30 & $57.8 \%$ & $69.6 \%$ & $16.9 \%(-13.6$ to 40.4$)$ & Not significant \\
\hline
\end{tabular}

*Abbreviations defined in glossary; RRR, NNT, and Cl calculated from data in article. 